\title{
Zur konkreten Utopie einer kritischen Universität
}

Wie wollen wir im akademischen Kontext arbeiten, in welchen Verhältnissen und zu welchen Themen? Unsere Vorschläge für eine gründliche Umgestaltung der Universität haben wir auf der Grundlage einer kritischen Bestandsaufnahme des Arbeitens und Lernens an deutschen Universitäten erarbeitet. Dies geschah im Rahmen einer von sub \urban initiierten Zukunftswerkstatt „Konkrete Utopie einer kritischen Wissensproduktion“, die am 30. April 2016 im Atelier des ISR[1] an der TU Berlin mit zehn Teilnehmer_innen stattfand. In diesem Text stellen wir die Ergebnisse der Zukunftswerkstatt vor.

\section{Warum haben wir eine Zukunftswerkstatt zur Entwicklung konkreter Utopien einer kritischen Universität durchgeführt?}

Immer wieder haben wir in der Vergangenheit festgestellt, dass es teilweise an utopischen Gegenentwürfen zu den derzeitigen Zuständen an Universitäten sowie insgesamt in Bezug auf die Produktion von Wissen im Rahmen wissenschaftlicher Forschung und Lehre fehlt. Zwar haben wir einerseits als Mitglieder des Wissenschaftssystems alle ein vielschichtiges und auf jahrelangen Erfahrungen basierendes Missbehagen an den gegenwärtigen Verhältnissen und können auch zahlreiche Aspekte nennen, die wir kritisieren. Andererseits jedoch fehlt uns sowohl eine kollektive Vision als auch eine Herangehensweise, die konkrete Veränderungen impliziert und hervorruft.

Aus diesem Grund haben wir uns entschieden, eine Zukunftswerkstatt durchzuführen. Damit folgen wir einem strukturierten Ansatz, der darauf beruht, gemeinsam sowohl Kritik zu üben als auch utopische Ideen und daraus resultierend konkrete Vorschläge für erste Schritte zur Veränderung des Wissenschaftssystems zu entwickeln. Der Ablauf von Zukunftswerkstätten ist entsprechend strukturiert: Auf eine Kritikphase folgt eine Phantasie- und Utopiephase sowie anschließend eine Umsetzungsphase. Unsere Zukunftswerkstatt war als Tagesworkshop zeitlich knapp angesetzt (oft wird für dieses Format zu 2-3 Tagen geraten). Die Ergebnisse des Workshops haben wir in einem gemeinsamen Schreibprozess weiterentwickelt. Damit verstehen wir den Entwurf einer konkreten Utopie nicht als ein bloßes imaginäres 
zukünftiges Bild einer kritischen Wissenspraxis, das dem der gegenwärtigen Praxis gegenüber steht, sondern sehen die einzelnen Prozesse der kollektiven Organisation und Arbeit sowie auch des Schreibens selbst als wichtige Formen eines reflexiven, dynamischen und offenen Denkens und Handelns, das mögliche Alternativen nicht nur kritisch diskutiert und entwickelt, sondern gleichzeitig auch kollektiv in der Gegenwart testet. Hinsichtlich der Zusammensetzung der Teilnehmer_innen war es uns ein Anliegen, unterschiedliche Perspektiven des akademischen Betriebs mit einzubeziehen: Studierende und studentische Mitarbeiter_innen, arbeitslose beziehungsweise prekär beschäftigte Akademiker_innen und solche mit stabileren universitären Beschäftigungsverhältnissen nahmen an der Werkstatt teil.

\section{Kritik: Der gegenwärtige Zerfall einer qualitativen und kriti- schen Wissenspraxis}

In der Kritikphase haben alle Teilnehmenden zunächst individuelle Kritikpunkte gesammelt, die im Anschluss drei thematischen Bereichen zugeordnet wurden:

1. Kritik am gegenwärtigen Umgang mit Inhalten in Lehre und Forschung

1. Kritik an Rahmen- und Arbeitsbedingungen im Wissenschaftssystem

1. Kritik an der Zurichtung der Subjekte im Wissenschaftssystem

\section{Kritik am gegenwärtigen Umgang mit Inhalten in Lehre und Forschung}

Seit einiger Zeit scheint es mehr um Quantität als um Qualität der an der Universität produzierten Inhalte zu gehen, bezogen sowohl auf Inhalte der Forschung als auch der Lehre. Dies betrifft die Menge der eingeworbenen Forschungsmittel ebenso wie die Anzahl der Publikationen bei beidem gilt: je mehr desto besser und nicht, je fundierter das Projekt oder die Publikation, desto besser. Damit verbunden ist ein hoher Druck, der auf den Wissenschaftler_innen lastet und der nicht selten dazu führt, dass die Beantragung von Forschungsförderung von der gewünschten Drittmittelquote bestimmt ist und weniger vom intendierten Erkenntnisgewinn - alles, was geht, wird wild beantragt.

Kritisiert wurde innerhalb der Gruppe zudem, dass es zunehmend um ,affirmative Inhalte' geht, dass oft kein echtes Wissensinteresse oder Interesse am Erkenntnisgewinn besteht und die Forschung in vielen Bereichen immer marktförmiger und verwertungsorientierter organisiert wird (die Logiken der freien Wirtschaft kapern die Uni). Entsprechend werden die Wissensinhalte ausgerichtet. In der Folge gibt es wenig Zeit, um eigene Perspektiven und neue Zugänge zu entwickeln. Hinzu kommt, dass sich das Hochschulpersonal immer weniger kritisch in gesellschaftspolitische Debatten einmischt, was sich auch in der inhaltlichen Ausrichtung in Lehre und Forschung niederschlägt.

Das gegenwärtige Streben vieler Universitäten nach Exzellenzförderung scheint diese Tendenzen noch zu beflügeln. Statt Inhalte kritisch und nach dem Rahmen gesellschaftlicher Notwendigkeit auszurichten, gilt es für viele Hochschulen, ,um jeden Preis' die richtigen Themenschwerpunkte und Kooperationen einzugehen., Qualitativ anspruchsvolle Lehre und Forschung 
wird so nicht im Sinne einer progressiven Wissenspraxis, sondern anhand eines Exzellenzbudgets gemessen.

Kritisiert wurden auch die engen Disziplingrenzen und damit verbundene Grabenkämpfe, weil sie interdisziplinäre wissenschaftliche Arbeit behindern und nicht anerkennen. Trotz allseits geforderter Interdisziplinarität scheinen die Praxis an den Hochschulen und die Förderpraxis von Geldgebern das Gegenteil zu zeigen.

Zudem wurde bemängelt, dass die Anerkennung ,guter ' Lehre abzunehmen scheint. Dies steht mit der Verschulung der Hochschul(aus)bildung in Bachelor- und Masterstudiengängen in Verbindung. Hinzu kommt ein stetig wachsender Massenbetrieb an den Hochschulen, innerhalb dessen die Zahl der Studierenden steigt, die der Lehrenden aber nicht. Allein die Anzahl der eingeschriebenen Studierenden bestimmt den Verteilungsschlüssel für die an die Hochschulen ausgezahlten Gelder für die Grundlehre, nicht hingegen die Anzahl der Lehrenden. Hieraus ergibt sich insofern ein Zusammenhang, als dass derzeit viele Entwicklungen in der deutschen Hochschullandschaft auf eine zunehmende Entkopplung von Lehre und Forschung hinauslaufen.

Auch personell hat dies Folgen: Mit der Schaffung von ,Lehrkräften für besondere Aufgaben' beziehungsweise ,Hochdeputatsstellen“ wird darauf hingewirkt, dass Teile der Hochschullehre von extra dafür eingestellten Lehrenden absolviert werden, die wiederum keine Zeit zum Forschen haben. Dadurch besteht zum Einen die Gefahr, dass das Wissen, das sie ,vermitteln', sich vom jeweils aktuellen Forschungsstand entfernt und darüber hinaus Wissen als etwas ,Feststehendes', ,Statisches' vermittelt wird, das kanonisiert ist und nicht selbst Gegenstand forschenden Lernens und Lehrens sein muss. Andererseits wird mit der verstärkten Einführung solcher Hochdeputatsstellen eine eigene Klasse innerhalb des Wissenschaftssystems geschaffen, die es schwer haben wird, innerhalb desselben weiter zu kommen, da für eigenständige Forschung schlicht Zeit und Ressourcen fehlen und die Entwicklung eines eigenen Forschungsprofils als Grundlage für eine weitere Beschäftigung gilt.

Kritik an den Rahmen- und Arbeitsbedingungen im Wissenschaftssystem

Die Rahmenbedingungen des Arbeitens in der Wissenschaft werden von besonders perfiden Hierarchiegebilden geprägt, die man als ,(neo)feudalistisch beziehungsweise ,nepotistisch ' bezeichnen kann, da nach wie vor eine starke Abhängigkeit der wissenschaftlichen Angestellten von den Professor_innen fortbesteht und reproduziert wird. Dies betrifft sowohl die Ausgestaltung der Arbeitszeit und die überlassenen Aufgaben als auch die Wahl der zu bearbeitenden Inhalte und nicht zuletzt die Personalpolitik. Die befristet eingestellten Wissenschaftlichen Mitarbeiter_innen sind in vielen Fällen auf das Wohlwollen der Professor_innen angewiesen, die nach oftmals intransparenten Maßstäben ihre Arbeitsverträge verlängern - oder eben nicht. In dieser Position liegt es für die befristet eingestellten Wissenschaftler_innen oft näher, einen guten Kontakt zu dem/der Professor_in zu halten, als für eine Verbesserung ihrer Arbeitsverhältnisse zu kämpfen und damit in einen Konflikt einzutreten.

In vielen anderen Arbeitsbereichen gültige personalrechtliche Normen sind im Wissenschaftsbereich nicht existent. Dass das bestehende System 
den Wissenschaftler_innen keine langfristige Perspektive bietet, resultiert in starker Verunsicherung der Einzelnen (,Regime der Angst') ebenso wie in fehlender Solidarität und extremer Konkurrenz zu Kolleg_innen anstelle von konstruktivem Austausch, kollegialer Zusammenarbeit und solidarischem Miteinander. Fehlende Kooperation zwischen den Wissenschaftler_innen ebenso wie ihre vergleichsweise spät erreichte Selbständigkeit, sowohl in inhaltlicher als auch finanzieller Hinsicht, sind dramatische und intendierte Folgen dieser Bedingungen in einem auf Wettbewerb basierenden System. Die durch kurze Vertragslaufzeiten bedingte fehlende Kontinuität in Lehre und Forschung ist ein unerwünschter Nebeneffekt, der jedoch hingenommen wird. Qualität in Forschung und Lehre ist hauptsächlich eine Frage des persönlichen und Gruppenengagements und keineswegs eine Selbstverständlichkeit.

Auch das ,teleologische Professionsnarrativ betrachten wir als Problem, da darin glaubhaft gemacht wird, dass es in der Wissenschaft ausschließlich den gerichteten Weg, vom Studierenden zum/zur Professor_in' gibt und nichts anderes. Dies passt zu der Entwicklung, dass in den vergangenen Jahren ein Großteil der früheren Akademischen Ratsstellen und Hochschuldozenturen gestrichen wurde, welche Wissenschaftler_innen unbefristete Stellen jenseits von Professuren boten. Aus unserer Sicht bedeutet dies einen ,Zwang zur Karriere', ein ,in or out ' in einem zugespitzten und intransparenten Wettbewerb, was wir entschieden kritisieren.

Im Bereich der Rahmenbedingungen ist auch die Austeritätspolitik zu kritisieren, von der Hochschulen ganz und gar nicht ausgenommen sind: Mehr Arbeit muss von weniger Mitarbeiter_innen bewerkstelligt werden. Hinzu kommt, dass immer mehr Verwaltungsaufgaben erzeugt werden, die, wie beispielsweise die Neukonzeption von Studiengängen oder die Selbstverwaltung von Geldern, zunehmend viel zu viel Zeit und Kräfte aufseiten der wissenschaftlich Tätigen bindet. Die so wachsenden und ungleich verteilten Bürokratiestrukturen prägen zunehmend den wissenschaftlichen Alltag und tragen entscheidend zur Verstetigung gegenwärtiger Machtverhältnisse zwischen Hochschulleitung, Professor_innen und Mittelbau bei.

\section{Kritik an der Zurichtung der Subjekte im Wissenschaftssystem}

Das Wissenschaftssystem, aber auch das Bildungssystem insgesamt, wirkt mit an einer Zurichtung der darin befindlichen Subjekte. Wettbewerb und Konkurrenz führen zunehmend zu einer Entsolidarisierung und Vereinzelung, dienicht nur den Einzelnen schadet, sondern sich auch auf die Gesellschaft sowie auf das produzierte Wissen auswirken. Wenn sich Wissenschaftler_innen nur noch auf Selbstoptimierung ausrichten, es aber gleichzeitig an der Befriedigung so grundlegender Bedürfnisse wie gutem Essen in den Hochschulkantinen, ausreichend Schlaf, Entspannung und einer gewissen Sicherheit hinsichtlich der eigenen Lebensperspektive mangelt, ist das hochgradig problematisch und erzeugt verständlicherweise Stress und Frustration.

Es hat zudem Folgen für die Entwicklung von qualitativ hochwertiger Forschung und Lehre, die unter einem konstanten Wechsel von Personal leidet. Dass es so viele,Wanderarbeiter_innen' gibt, die von Jahr zu Jahr auf befristeten und Teilzeitstellen in teils verschiedenen Städten arbeiten, nur 
um irgendwie überhaupt im Wissenschaftssystem Fuß zu fassen, ist kaum anders zu verstehen als durch die nach wie vor existierende Leidenschaft für wissenschaftliche Wissensproduktion und -vermittlung sowie die zu geringe Zahl an gut bezahlten, unbefristeten und interessanten Arbeitsstellen in der Wissenschaft. Dass von Wissenschaftlichen Mitarbeiter_innen dann trotz ihrer Teilzeit- und Befristungslage erwartet wird, sich stark mit den Arbeitsinhalten und der jeweiligen Forschungs- und Lehrinstitution zu identifizieren, ist obendrein absurd.

Uns fiel hier auch der fehlende Gestaltungswille der Beteiligten auf, insbesondere der innerhalb des Systems strukturell Bessergestellten, den Professor_innen. Die meisten, selbst durch das System produzierte Einzelkämpfer_innen, die Konkurrenz und Wettbewerb internalisiert haben, richten ihren Fokus viel zu selten auf Veränderungsmöglichkeiten innerhalb des Systems. Dies führt dazu, dass sie ihren, auch kollektiv auszuübenden, relativ großen Einfluss und ihre zahlreichen Spielräume unterschätzen, nicht ausloten und nicht nutzen.

Kritisiert haben wir außerdem, dass an Hochschulen nach wie vor ein Mittelschichtshabitus gefördert wird, dem sowohl Klassismen, Rassismen und Sexismus zueigen sind als auch ein Obrigkeitsdenken. Davon betroffen sind auch Studierende, die nach wie vor aus recht homogenen sozialen Verhältnissen stammen, was sicherlich sowohl mit der Art des Wissens, das an Hochschulen vorausgesetzt und vermittelt wird, als auch mit Verhaltenserwartungen zusammenhängt.

Damit verbunden haben wir auch den gesteigerten ,Leistungsgeist ${ }^{\prime}$ der Studierenden kritisiert, die nicht mehr ausreichend kritisch hinterfragen, was von ihnen erwartet wird und stattdessen blind die gesellschaftlich gesetzten Leistungserwartungen versuchen zu erfüllen, ohne dabei über den Tellerrand zu blicken. Nicht selten kommen die Forderungen nach zunehmender Formalisierung und Verschulung der Lehr- und Prüfungsformen dabei aus den Reihen der Studierenden selbst.

Es wird deutlich, dass diese drei Bereiche unserer Kritik am Wissenschaftssystem ineinander greifen. So sind zum Beispiel der zunehmende Publikationsdruck und die Ausrichtung auf Quantität auch damit zu erklären, dass materielle Rahmenbedingungen durch Stellenbefristungen, Teilzeitstellen und die Ausrichtung der Forschung auf Drittmitteleinwerbung verschärft wurden. Dies wiederum erzeugt eine Einzelkämpfermentalität, zunehmenden Konkurrenzdruck sowie eine allgemeine Beschleunigung des wissenschaftlichen Alltags, der die Überlastung der in der Wissenschaft und Lehre tätigen Subjekte impliziert.

\section{Utopie: Eine andere Wissenspraxis ist möglich!}

Es muss nichts so bleiben, wie es ist. Wir sind davon überzeugt, dass es ,nur Utopien - also die Fähigkeit, progressive Ideen, Träume, Phantasien kollektiv zu denken und zu artikulieren - braucht, um die Verhältnisse zu verändern sowie alltägliche Dinge zu re-politisieren und in neue Richtungen zu treiben. Daher haben wir damit begonnen, uns gemeinsam eine kritische Universität und Wissenspraxis der Zukunft vorzustellen. Unser Material waren Papier und Stifte. Dabei herausgekommen sind drei Entwürfe, die vieles von dem 
adressieren, was wir in der vorangegangenen Phase kritisiert haben. Dabei wurde deutlich, dass wir uns keinesfalls darüber einig waren und sind, ob eine andere Wissenspraxis in den bestehenden wissenschaftlichen Institutionen möglich sein kann oder ob es Neugründungen außerhalb dieser geben muss.

1. Das erste Modell, eine Art Kugel-Zickzack-Netzmodell, ist aus der Vorstellung einer räumlichen Anordnung entstanden: einer runden Grundanordnung, die für Hierarchielosigkeit steht. Die Basis dieses utopischen Modells bilden Neugier, Offenheit, Großherzigkeit und Großzügigkeit, Kollektivität und Solidarität. Wissen ist in diesem recht umfassenden Arbeits-, Lern- und Lebensmodell nicht hierarchisiert; der Wissensbegriff ist erweitert. Die Trennung von Schule und Hochschule ist aufgehoben, ebenso die Trennung von abstraktem und praktischem Wissen sowie die Trennung von Wissens- und Reproduktionsprozessen. Unterschiedlichste Wissens- und Praxisbereiche sind im Kugel-Zickzack-Netzmodell integriert. Personen verschiedenen Alters und mit unterschiedlichen Befähigungen, darunter auch Kinder, tragen die Forschungs-, Lehr- und Lernprozesse. Der Prozess der Wissensproduktion ist undeterminiert und alle Involvierten werden als Expert_innen angesehen. Eine Art forschendes Lernen wird praktiziert, bei dem Forschen und Lernen eng verkoppelt sind. Es wird keine rigide Spezialisierung erwartet. Verschiedene Zeithorizonte der Teilnahme sind möglich. Nicht nur das Tun steht im Vordergrund; verschiedene Geschwindigkeiten sind möglich. Es gibt genügend Raum und Zeit für Muße, Zuschauen und Langsamkeit. Das Kugel-Zickzack-Netzmodell basiert auf einem pluralistischen Ansatz und demokratischen und solidarischen Prozessen. Politische Mobilisierung ist ein wichtiges Anliegen. Das Gebilde ist selbstorganisiert und autonom. Dabei existiert eine prinzipielle Gestaltbarkeit von Inhalten und Umgebung, die immer verhandelbar sind. Der Alltag in diesem Modell ist von räumlichen und inhaltlichen Aneignungen geprägt. Das Ganze wird von einem positiven Menschenbild getragen, das auf Vertrauen und Kooperation beruht. Die Beteiligten bekommen ein auskömmliches und bedarfsgerechtes Grundeinkommen. Dieses lokal verwirklichte Gebilde ist mit vielen anderen, ähnlichen und andersartigen Gebilden vernetzt und verkoppelt.

2. Das zweite Modell fokussiert mit seiner „Peer-to-Peer University“ eine radikale Verbesserung der Arbeitsbedingungen und wissenschaftlichen Inhalte an Universitäten. Universitäten werden dabei als diejenigen Orte (um-)gedacht, an denen kritische Reflexion ebenso wie kritisches Praxiswissen hergestellt wird. Die Lehre, Studiengänge und Forschung mit wechselnden Inhalten sind durch Lehrende und Studierende selbstorganisiert und werden unabhängig von wirtschaftlichen Interessen und Karrierekriterien entwickelt. Um aber einer Beliebigkeit der Inhalte vorzubauen, findet eine kollektive Relevanzbestimmung bezüglich der Inhalte und Themen von Lehre und Forschung statt, auch zusammen mit wissenschaftsexternen Akteur_innen wie beispielsweise Gewerkschaften und sozialen Bewegungen. Der dafür notwendige Austauschprozess stellt die gesellschaftliche und wissenschaftliche Relevanz wissenschaftlicher Arbeit immer wieder neu her. Um diesen wichtigen Austausch zu 
gewährleisten, existieren frühzeitig langfristige Arbeitsperspektiven. Eine Hierarchisierung der Lehrenden ist in diesem Modell nicht vorgesehen, alle sind forschende, lehrende und verwaltende Mitarbeiter_innen.

3. Das dritte Modell zeichnet einen Entwurf für die Freie Autonome Uni Kreuzberg (FUK; wird jährlich umbenannt). Sie ist eine ,open university', die zugänglich ist für (fast) alle. Da nicht jede_r mitmachen darf, sind auch Konflikte vorprogrammiert, die aber willkommen sind. Die Uni ist selbstverwaltet. Es gibt ein bedarfsorientiertes Einkommensmodell und keine Abstufung ,studentischer Löhne. Außerdem werden Stipendien vergeben und die Kinderbetreuung ist organisiert. Die FUK ist in einem Hochhaus in Kreuzberg angesiedelt. Bei der Suche nach nutzbaren Räumlichkeiten fiel ins Auge, dass in guter 1968er Tradition eine Umnutzung des Axel-Springer-Hochhauses sinnvoll wäre. Dieses wurde daraufhin gekapert. Im oberen Teil des Gebäudes wird mehrsprachig geforscht und gelehrt; im unteren Bereich finden sich Ruhe- und Toberäume, Küchen und Kantinen, eine Druckerei und Werkstätten sowie die Bibliothek. Im Außenbereich gibt es einen Garten. Professor innen gibt es nicht, stattdessen Lernkollektive, Forschungskollektive und Kochkolletive. Die FUK ist überregional vernetzt mit offenen Unis, die in anderen Städten entstanden sind. Das an der FUK generierte Wissen ist irrelevant und tribal. Ansonsten ist die FUK entsprechend größtmöglicher Intransparenz organisiert. Der Entortung und Entfremdung setzt sie soziale Rückbindung entgegen.

Die Ideen aus den drei entworfenen Modellen haben wir in vier Themenblöcke sortiert:

1. Offenheit/Zugang zu Wissen; gleichzeitig geschützter Raum

2. Formen der räumlichen Organisation

3. Kritische Wissenspraxis, Formen der institutionellen Organisation

4. Relevanz von Wissen, Formen der Wissensproduktion, Wissensformen

Nachdem wir mit diesem Schritt unsere kollektiven Prioritäten gesetzt hatten, haben wir uns in der Umsetzungsphase zunächst mit den zwei Bereichen ,kritische Wissenspraxis‘ und ,Relevanz von Wissen` beschäftigt.

\section{Umsetzung: Wege zu einer kritischen Universität}

\section{Kritische Wissenspraxis}

Kritik ist eine Praxis, die von solidarischem Handeln geprägt ist und kein Selbstzweck. Eine kritische Wissenspraxis kann sich nur in einem von Neugier geprägten Klima entfalten, in dem es freie Denkräume gibt und Verteidigung oder Rechtfertigungen von Interessen außen vor bleiben. Weitere Voraussetzungen sind die Zugänglichkeit, bezogen auf räumliche, finanzielle und habituelle Aspekte der Wissenspraxis, sowie auf die Selbstbestimmung bei gleichzeitiger Anerkennung des Grundsatzes der politischen Gleichheit aller. Autonomie, Öffentlichkeit und Selbstverwaltung fördern die Entwicklung einer kritischen Wissenspraxis wohingegen weniger klar ist, ob ein räumlich 
dezentraler Ansatz, in dem eine solche Wissenspraxis grundsätzlich überall stattfinden kann, gegenüber neuen Institutionen vorzuzuziehen ist.

\section{Relevanz von Wissen}

Was ist Wissen? Welche Relevanz hat welches Wissen? Hier sind mehr Diskussions- und Reflexionsprozesse nötig, um hegemoniale Wissensproduktion zu kritisieren. Lehren und Lernen gehen ineinander auf, daher müssen Wissensproduktion und -weitergabe verstärkt in verkoppelten Lehr-Forschungs-Kontexten stattfinden. Dies ermöglicht kollektive Prozesse der Relevanzzuschreibung und -verhinderung in Bezug auf die Wissensproduktion. Wir haben hier noch kein passendes Vokabular entwickelt, um die verschränkten Lehr-Forschungs-Kontexte zu beschreiben, in denen sich die Dualität und Hierarchisierung von Forschen (für Expert_innen) und Lehren (als Wissensvermittlung, bei der die Idee des Eintrichterns stark mitschwingt) auflöst zugunsten einer Anerkennung, dass sowohl Lehren als auch Forschen gleichzeitig Lernen und Wissensreflexion bedeuten.

Immer wieder haben wir uns gefragt, warum die Strukturen in der Wissenschaft so statisch scheinen, und haben uns noch einmal mit der fehlenden Solidarität unter den Kolleg_innen beschäftigt, insbesondere unter Professor_innen. Es scheinen viel mehr Gestaltungsspielräume zu existieren, als Professor_innen und Dekanate von Fakultäten gegenwärtig wahrnehmen. Sie müssen viel stärker als bisher entdeckt und genutzt werden. Eine Form des Umgangs mit unzumutbarem Sparzwang und schlechten Arbeitsbedingungen, insbesondere der unteren Statusgruppen, wäre eine stärkere Nutzung von Verweigerungsmöglichkeiten. STOPP sagen, individuell und kollektiv. Dies setzt ein gewisses Rückgrat der Professor_innen voraus, die sich auf dem individuellen Einzelkämpferweg zu ihrer Professur womöglich immer wieder korrumpieren lassen mussten und vermutlich eher weniger kritisches Potenzial haben als noch die Angestellten im Mittelbau, weil sie sich sonst auf dem Weg in die Institution selbst im Wege gestanden hätten. Solidarität wieder zu lernen, könnte daher ein sinnvoller Weg für Professor_innen sein, um kollektives Handeln und gegenseitiges Vertrauen weiterzuentwickeln. Dies bedeutet am Ende, dass die Professor_innen die bedürftigsten und zugleich mächtigsten Personen im Wissenschaftsapparat sind.

Vonseiten der teils noch solidarisch agierenden Studierenden und der Beschäftigten im Mittelbau könnte daher Empathie für die Mitglieder der Statusgruppe der Professor_innen ein Weg sein, um sich der kollektiven Gestaltungsspielräume bewusst zu werden und ein kollektives Handeln weiterzuentwickeln. Doch auch mit der Solidarität der Beschäftigten im Mittelbau ist es nicht weit her. Kürzere Vertragslaufzeiten, geringere Stellenanteile und eine strikt vollzogene Spaltung zwischen jenen auf Haushaltsstellen, die Forschung und Lehre betreiben, sowie anderen, die sich auf Drittmittelstellen alleinig auf die Forschung konzentrieren (und vom Betrieb an der Universität nur einen Ausschnitt mitbekommen) resultieren in einer wachsenden Fragmentierung im Mittelbau. Bei den Studierenden sind ähnliche Prozesse der Entsolidarisierung zu beobachten, ebenso getrieben von Prozessen der Selbstoptimierung und der Arbeit am eigenen Lebenslauf mittels Einhaltung der vorgeschriebenen Studiendauer, entsprechender Auslandsaufenthalte 
und möglichst ausgeprägten Praktika sowie von einer zunehmend empfundenen Unsicherheit bezüglich Beschäftigungsaussichten nach dem Studium.

Ein von Hierarchien befreites Miteinander scheint an den Universitäten vonnöten, welches allerdings erst erarbeitet werden muss. Auch Arbeitsbedingungen auf Augenhöhe - unbefristet, angemessen bezahlt für alle sind dazu ein sinnvoller Weg. Denn Wissenschaftliche und Studentische Mitarbeiter_innen können auch aufgrund befristeter Verträge gegenüber Universitäten kaum Loyalität entwickeln. Studierende durch selbstorganisierte und autonome Lehr- und Lernformate aktiver in die Wissenspraxis einzubeziehen und diese zu unterstützen, so wie es andere Universitäten in Ansätzen bereits verfolgen (zum Beispiel das Projekt „Interflugs“an der UDK Berlin oder freie Universitäten wie die „Parallel School“, „Open University“, „Silent University“ et cetera), könnten zudem stärkere Synergien zwischen Lehrenden und Lernenden generieren, um der zunehmenden De-Politisierung und dem blinden beziehungsweise konformistischen Leistungswahn der gegenwärtigen Wissenspraxis entschieden entgegen zu wirken.

\section{Endnoten}

[1] Der Ort, an dem wir im April 2016 unsere Zukunftswerkstatt veranstaltet haben, war nur aufgrund von utopischem Denken und innerinstitutionellen Kämpfen in dieser Weise für uns nutzbar: Das ,Atelier` des Instituts für Stadt- und Regionalplanung (ISR) an der TU Berlin ist erst seit Herbst 2015 durch das ISR nutzbar; erst nach jahrelangen Kämpfen mit der Raumvergabestelle der TU wurde es dem Institut durch die Verwaltung ermöglicht, hier die im Rahmen des städtebaulichen Entwurfs dringend benötigten studentischen Arbeitsplätze anzubieten. Dies zeigt: Wo - im Universitätssystem - ein Wille ist, ist auch ein Weg.

\section{Autor_innen}

Die Teilnehmer_innen der Zukunftswerkstatt „Konkrete Utopie einer kritischen Wissensproduktion“ sind Studierende und studentische Mitarbeiter_innen, arbeitslose bzw. prekär beschäftigte Akademiker_innen und welche mit stabileren Beschäftigungsverhältnissen an der Universität.

info@zeitschrift-suburban.de 